\title{
PROBABILITY DENSITY EVOLUTION FOR TIME-VARYING RELIABILITY ASSESSMENT OF WING STRUCTURES
}

\author{
Sajad SARAYGORD AFSHARI (D), Seid H. POURTAKDOUST (D)* \\ Center for Research and Development in Space Science and Technology, \\ Sharif University of Technology, Tehran, Iran
}

Received 11 July 2017; accepted 23 May 2018

\begin{abstract}
Reliability evaluation is a key factor in serviceability and safety analysis of air vehicles. Structural health monitoring methods have grown to a degree of maturity in many industries. However, there is a challenging interest to tie in SHM with reliability assessment. In this respect, consideration of stochastic structural dynamics with SHM data and random loadings opens a new chapter in failure prevention. The current study focuses on the stochastic behavior of structures as a way to relate SHM data with reliability. In this respect, uncertain factors such as atmospheric turbulence, structural parameters, and sensor outputs are considered in the process of reliability assessment. Firstly, an experimental evaluation is conducted using a simple cantilevered beam. Subsequently, system identification is weaved in with a probability density evolution equation for calculating the reliability of a wing structural component. Numerical simulations demonstrate that structural reliability of a typical WSC can be effectively evaluated. The proposed scheme paves the way for new SHM research topics such as online life prediction and reliability based failure prevention.
\end{abstract}

Keywords: system identification, reliability assessment, stochastic loadings, online SHM, structural health monitoring, life rediction.

\section{Introduction}

Many engineers have focused on aircraft Structural Health Monitoring (SHM) for its potential cost savings in lieu of structural related failures. Advances in the area of aircraft SHM are mostly due to efforts devoted to the developments of appropriate software and hardware packages that provide accurate data from structural physics as well as external loads. However, high-performance necessities of modern aircrafts still face new challenges in design for safety and maintainability of the vehicle lifecycle. Besides, new requirements for improved safety, reduced weight and low-cost maintenance, signals provisions for novel failure preventive maintenance and inspection routines as well as enhanced design and manufacturing process. A key factor influencing the maintenance cost is enhanced reliability that is directly related to aircraft life extension. Reliability Assessment (RA) can be conducted from conceptual and preliminary design phases to prototyping, testing, and even during the operational phase of an aircraft life cycle. The concept of SHM-based RA, which can be conducted during the operational phase of the life cycle, allows for reduced operational and maintenance costs to airlines.
The latter has instigated the current research in an effort to utilize SHM data for online structural reliability assessment. The current authors have recently evaluated this concept via experiments on a wing section (Saraygord Afshari \& Pourtakdoust, 2017); here the concept is extended to a general aircraft wing via numerical simulations.

Recently, Due to the increasing use of composite materials for weight reduction in aircraft wings, damages are becoming more crucial throughout the wing structures (Grondel, Assaad, Delebarre, \& Moulin, 2004). Statistical data show that delamination and disbonds between stiffeners and composite skins are one of the most important damage mechanisms for composite wing type structures. This latter damage type can be frequently linked to dynamic loads acting on the wing structure when the aircraft is in service (Grondel et al., 2004). Generally, schemes of finding potential failures via dynamic response of structures are known as vibration-based SHM methods (Trendafilova, Cartmell, \& Ostachowicz, 2008), and they are categorized under global nondestructive testing (NDT) methodologies. SHM techniques can be classified into four main groups that include those concentrating

${ }^{\star}$ Corresponding author. E-mail: pourtak@sharif.edu 
on basic statistical analysis (Mustapha, Manson, Pierce, \& Worden, 2005), techniques based on time series analysis (Fassois \& Sakellariou, 2009), frequency domain (modal) analysis schemes (Belisario-Briceno, Zedek, Camps, François, Escriba, \& Fourniols, 2014) and time-frequency analysis techniques (Raghavan \& Cesnik, 2007).

Even though practicality of the SHM for structural safety is proven in the literature, its usefulness can be significantly enhanced when aggregated with reliability considerations. There exists a key distinction between the structural component reliability and that of a technical part such as valves, pumps, generators, transistors, etc. Structural failures not only occur due to the aging process but also due to simultaneous interactions between the aging process and extreme events (Tuegel et al., 2013). Considering uncertainties in design loads and material strength, aircraft structural reliability can be estimated before the operation as well. However, aircraft system and structural reliability could degrade in flight due to some unforeseen events that could lead to eventual failures. In this respect, online structural RA could be extremely valuable toward enhanced safety and catastrophic failure avoidance. A practical way to observe structural system reliability is the detection of potential damages leading to eventual failures. In this respect, Yao et al. proposed a holistic view in which health monitoring, damage detection, and reliability evaluation are defined as sequential components of a value chain (Zhang, $\mathrm{Xu}, \& \mathrm{Li}, 2011$ ).

Aiming towards estimation of remaining life and reliability via structural response and SHM techniques, a suitable reliability approach needs to be selected as well. SHM data have been studied together with RA methods previously in different approaches. However, most of them are based on quantification of safety with respect to extreme events produced by the load effects (Okasha, Frangopol, Saydam, \& Salvino, 2011; Soliman, Barone, \& Frangopol, 2015). In these approaches, having a proper knowledge about the extreme value distribution is of vital importance. Another way to enhance SHM data with reliability interpretation is the concept of fatigue reliability (Soliman et al., 2015). In the fatigue reliability approach, the probability of being in each operational phase is decisive and it should be considered. Variability, statistical uncertainty, model uncertainty, measurement noise, and errors that influence damage modeling encourages one to develop a stochastic framework to incorporate these uncertainties in life prediction assessment (Zhu, Huang, Peng, Wang, \& Mahadevan, 2016). In 2004, Li and Chen presented a Probability Density Evolution (PDE) equation according to the principle of preservation of probability (Li \& Chen, 2004) that allowed for randomness propagation in dynamical systems. Via the method of PDE equation, there is no need for additional information about the PDF of events and operational condition, since the PDE equation continuously updates the PDFs. Zhang et al., utilized the PDE equation together with system identification techniques in order to evaluate the reliability of a stochastic building structure (Zhang et al., 2011).
Applying PDE equation, the current study is aimed towards the utility of SHM data for real-time reliability analysis of aircraft wing structures under system and load uncertainties. Considering the fact that most SHM techniques will only provide health information without sufficient interpretation or prediction capabilities, an emphasis is made to present an integrated technique that assesses the wing structural reliability without any need for additional information about the PDFs of events and structural parameters. For this purpose, initially, a set of piezoelectric sensors are assumed to be distributed on a typical Wing Structural Component (WSC) that provide the data about structural deformations emanating due to stochastic atmospheric disturbances. Subsequently, a stochastic system identification is utilized to determine the failure probability density function, which is the foundation for proposed reliability evaluation method. In this sense, clear air turbulence (CAT) is considered as a continuous stochastic gust model via the Dryden model with randomly varying intensity. Numerical simulation is performed on a clamped WSC under different damage scenarios. Additionally, the WSC physical structural parameters are also taken as random variables in order to achieve a full stochastic evaluation of system dynamics as a way for a better reliability analysis, which is not seen in the literature for such a problem.

The remaining organization of this paper is as follows: the basic methodology of probability density evolution function for reliability calculation and its experimental evaluation is described in section two. Section three is devoted to a description of the WSC geometry, its preparation for finite element method (FEM) analysis, and SHM data simulation. Continues gust modeling and the resulting random load power spectral density (PSD) derivation are described in section four. Finally, section five deals with the reliability computation and simulation process and the results, followed by concluding remarks and future research directives in section seven.

\section{PDE equation for online reliability assessment}

The current study utilizes the probability of measured dynamic response to determine the WSC reliability. In this regard, a damage detection method based on statistical moments of the physical parameters (Xu, Zhang, Li, \& $\mathrm{Xia}, 2009$; Zhang, Xu, Xia, \& Li, 2008), is employed together with PDE equation to find the probability of safe operation based on structural response (Li \& Chen, 2004; Saraygord Afshari \& Pourtakdoust, 2018). This method is implemented for a WSC via a single limit state function as well as the work recently published in (Raouf \& Pourtakdoust, 2017). In this sense, governing equations for PDE, system identification, and their combination for WSC reliability assessment are described in this chapter.

\subsection{The governing equations for WSC reliability analysis}

Without loss of generality, a wing structure can be discretized into a multi-degree of freedom structure with the 
following equation of motion in matrix form (Xu et al., 2009)

$$
\boldsymbol{M} \ddot{\boldsymbol{X}}(t)+\boldsymbol{C}(\Theta) \dot{\boldsymbol{X}}(t)+\boldsymbol{K}(\Theta) \boldsymbol{X}(t)=g(\Psi, t),
$$

where $\ddot{\boldsymbol{X}} . \dot{\mathbf{X}} . \mathbf{X}$ are the acceleration, velocity and displacement vector of $\mathrm{N}$ order; $\mathrm{g}(\Psi, t)$ is a random or deterministic external excitation; $\Psi$ is the random parameter vector with known probability density function; $p_{\Psi}(\psi)$ reflecting the uncertainty in the excitation intensity. $\mathrm{M}, \mathrm{C}$ and $\mathrm{K}$ are the $\mathrm{N} \times \mathrm{N}$ mass, damping and stiffness matrices respectively. Here, $\Theta$ signifies the random parameter vector related to the physical uncertainties with known probability density function $p_{\Theta}(\Theta)$. All random parameters can be combined in $\mathrm{Z}$, where $\mathbf{Z}=\mathbf{Z}(\Theta, \Psi)$.

Further, to evaluate the reliability of the stochastic WSC, the total random parameters, $\mathrm{Z}$ is discretized at representative points $\mathbf{z}_{\mathbf{i}}=\left(\theta_{\mathrm{q}}, \psi_{\mathrm{r}}\right)$. where $\mathrm{i}, \mathrm{q}$ and $\mathrm{r}$ are integer values from one to $N_{z} \cdot N_{\theta}$ and $N_{\psi}$ respectively. For each random value of $\Theta \cdot \theta_{q}\left(q=1,2, \ldots, N_{\theta}\right)$. The structural stiffness parameters, can be identified via the stochastic system identification method (Xu et al., 2009; Zhang et al., 2008). For this purpose, a WSC with N shear ribs as shown in Figure 1 is considered and the external excitation $\mathrm{g}(\Psi, t)$ produces structural vibrations whose displacements responses are measured for reliability assessment.

For each random $\theta_{\mathrm{q}}$, the theoretical fourth-order moment vector of the rib displacements is denoted by $\mathrm{M}_{4 \theta \mathrm{q}}$ (k) and it is computed for each rib displacement $\left(\Delta x_{i}\right)$ as (Xu et al., 2009),

$$
M_{4 \theta i}=3 \sigma_{\Delta x_{i}}^{4}=3\left\{\begin{array}{l}
\int_{-\infty}^{\infty}\left[\sum_{k=1}^{N} \Delta \alpha_{i k}(\omega) C_{k}(\omega)\right] \times \\
{\left[\sum_{k=1}^{N} \Delta \alpha_{i k}(\omega)^{*} C_{k}^{*}(\omega)\right] d \omega}
\end{array}\right\},
$$

where $C_{k}(\omega)$ is the Fourier transform of $\mathrm{fk}(\mathrm{t})$ and

$$
\alpha_{i k}(\omega)=\sum_{j=1}^{N} \frac{\phi_{i j}(\boldsymbol{K}) \cdot \phi_{k j}(\boldsymbol{K})}{\omega_{j}(\boldsymbol{K})^{2}-\omega^{2}+2 i \omega \omega_{j}(\boldsymbol{K}) \xi_{j}},
$$

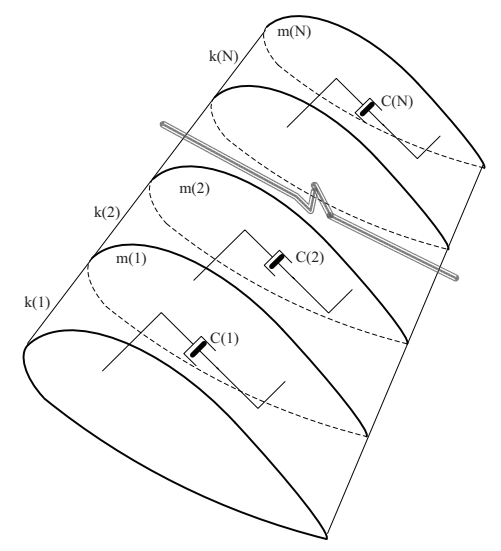

Figure 1. An N-ribs shear aircraft wing model where, $\Delta \alpha_{i k}(\omega)^{*}$ and $C_{k}^{*}(\omega)$ are the conjugates of $\Delta \alpha_{i k}(\omega)$ and $C_{k}(\omega)$ respectively. On the other hand, the measured fourth-order moment vector of the rib displacements is directly calculated from the displacement responses recorded by the SHM system, and denoted as $\hat{\mathbf{M}}_{4}$. Hence, the residual vector between the theoretical and experimental fourth order moments at each instant of time will be:

$$
\boldsymbol{F}_{\theta}(\boldsymbol{k})=\boldsymbol{M}_{4 \theta_{q}}(\boldsymbol{k})-\hat{\boldsymbol{M}}_{4} .
$$

At this point, system identification of the undamaged or damaged WSC can be performed by interpreting the problem as a Model-in-the-Loop-Optimization (MILO) problem and minimizing a cost function such as $\|\mathbf{F}(\mathbf{k})\|^{2}$. The output of this optimization is the rib equivalent stiffness parameter. In this regard, a Tabu Continuous Ant Colony System (TCACS) together with MILO is utilized for best optimization performance. Further details about the above mentioned method is presented in (Nobahari, Kordkheili, \& Afshari, 2014). It should be noted that due to random nature of $\Theta$. The identified structural stiffness is also random and referred to as $\mathbf{k}_{\Theta}(\boldsymbol{\theta})$.

Next, the WSC reliability under dynamic excitation $\mathrm{g}(\Psi, t)$ can be evaluated via,

$$
R(t)=P\left\{X(\tau) \in \Omega_{s} . \tau \in[0 . t]\right\},
$$

where $\mathrm{P}$ is the probability of the random event and $\Omega_{\mathrm{S}}$ reflects the safe operational domain for desired measured/ estimated variable. Equation 5 indicates that the WSC reliability is related to the probability of the random event being in the safe domain of the operational flight time duration $[0, \mathrm{t}]$.

Considering the method of PDE equation ( $\mathrm{Li}, 2016)$, the joint $\mathrm{PDF}$ of $\mathrm{X}(\mathrm{t})$ and the random parameter vector $\mathrm{Z}$, can be calculated via following evolutionary equation $(\mathrm{Li}$, 2016; Zhang et al., 2011):

$$
\frac{\partial p_{X Z}(x, \boldsymbol{z}, t)}{\partial t}+\dot{\boldsymbol{X}}(\boldsymbol{z}, t) \frac{\partial p_{X Z}(x, \boldsymbol{z}, t)}{\partial x}=0
$$

with the initial condition specified by,

$$
\left.\partial p_{X Z}(x, \boldsymbol{z}, t)\right|_{t=t_{0}}=\delta\left(x-x_{0}\right) p_{z}(\boldsymbol{z})
$$

the solution of the above equation represents the joint PDF of the structural physical parameters as well as its response, $\breve{p}_{\mathrm{XZ}}(x, \boldsymbol{z}, t)$, accordingly, the PDF of probable structural response can be calculated via its integration over the parameter domain of variation.

$$
\breve{p}_{X}(x . t)=\int_{\Omega_{z}} \breve{p}_{X Z}(x, z, t) d z
$$

and the structural component reliability will be given by:

$$
R(t)=\int_{\Omega_{s}} \breve{p}_{X}(x, t) d x,
$$

where $\Omega_{\mathrm{S}}$ is the safe domain. For the symmetrical double boundary problem, Eq. (9) becomes,

$$
R(t)=\int_{-x_{b}}^{x_{b}} \breve{p}_{X}(x, t) d x
$$




\subsection{Numerical algorithm for online WSC reliability assessment}

The basic flowchart suggested for the assessment of the reliability of a WSC is presented in Figure 2 and described as follows:

Discretize stochastic variation of $\mathrm{Z}$ at representative points $\mathrm{Z}_{i}=\left(\theta_{\mathrm{q}}, \psi_{\mathrm{r}}\right)$, in the domain $\Omega_{\mathrm{Z}}$.

For each structural random parameter, identify the stiffness parameters of the structural component, $\mathrm{k}_{\Theta}\left(\theta_{\mathrm{q}}\right)$.

For a given $\mathrm{z}_{i}=(\theta \mathrm{q}, \psi \mathrm{r})$ and its corresponding identified stiffness vector $\mathrm{k}_{\Theta}\left(\theta_{\mathrm{q}}\right)$, calculate the velocity $\dot{\mathbf{X}}\left(\mathbf{z}_{i}, t\right)$ by solving Eq. (1) using a deterministic numerical method;

Substitute $\dot{\mathbf{X}}\left(\mathbf{z}_{i}, t\right)$ into Eq. (6) and solve the differential equation.

Repeat Steps 3 and 4 to determine $\breve{p}_{\mathrm{XZ}}\left(x, \boldsymbol{z}_{\boldsymbol{i}}, t\right)$ at every discrete points.

Utilize the results in Step 5 to obtain $\breve{p}_{\mathrm{X}}(x, t)$ through the discretized version of Eq. (8):

$$
\breve{p}_{X}(x, t)=\sum_{i=1}^{N_{i}} \breve{p}_{X Z}\left(x, z_{i}, t\right) \text {. }
$$

Carry out the numerical integration in Eq. (10) to obtain the structural reliability with respect to time.

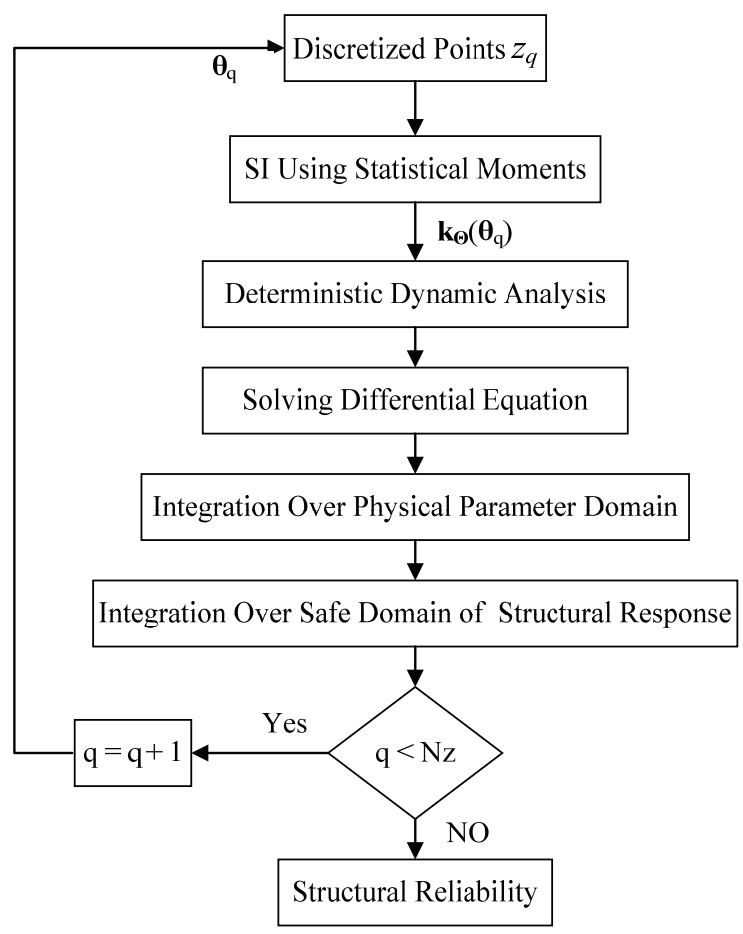

Figure 2. Numerical Flowchart for the WSC reliability analysis

\subsection{Experimental evaluation of the reliability assessment method}

As the reliability is an important index that always deals with safety and risk analysis, it is vital to evaluate the efficiency and accuracy of the presented reliability evaluation method. Here, a cantilevered beam is chosen as the simplest structure that can be used for evaluating the presented reliability calculation method. In the current study, loading and test fixtures were designed in a way to accelerate specific failure modes while replicating field performance.

\subsubsection{Experimental Setup and test characterization}

In order to assess the effectiveness and accuracy of the presented RA method, it is required to define proper failure modes, loadings, and progressive damage type. In this work, a cantilevered Twintex ${ }^{\oplus}$ fabric composite beam at room temperature pre-pregged with $60 \%$ fiber volume content $(\mathrm{E}=20 \mathrm{GPa}, \rho=1197 \mathrm{Kg} / \mathrm{m} 3)$, is used for the experimental AVC setup. a piezoelectric actuator is attached to the beam for exciting the beam and a piezoceramic sensor is also bonded at the cantilevered end of the beam for strain sensing. The beam and piezoelectric patches thicknesses are $2 \mathrm{~mm}$ and $0.6 \mathrm{~mm}$, respectively. Other dimensions are given in Figure 3 and the experimental setup is demonstrated in Figure 4. It is intuitive that in the presence of damage, the local stiffness will decrease and this reduction should be seen in the beam's structural response under excitation. Here, for structural reliability assessment during external loadings, a sinusoidal excitation force with random frequency and intensity is exerted to the damaged structure in a time interval of 120 seconds. Depending on the excitation force and physical parameters uncertainties, structure fails at different times during the accelerated test procedure. Concluded from some pre-experimental investigations, a sensor feedback in the domain of $[-3.2,3.2]$ Volts was chosen as a safe domain of performance for the current system to increases the chance of failure during the accelerated test.

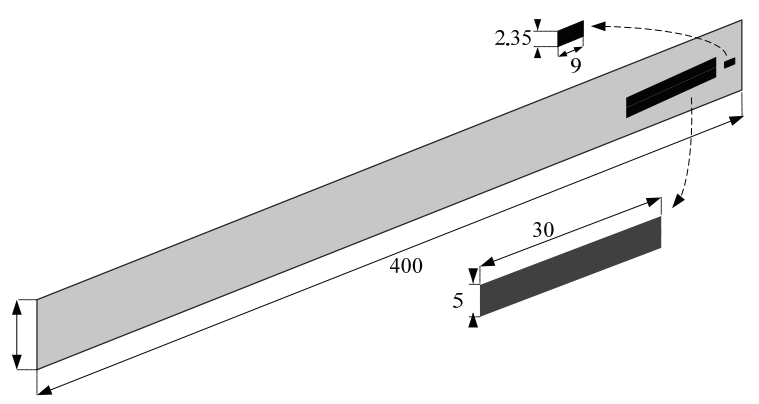

Figure 3. Experimental model dimensions (mm)

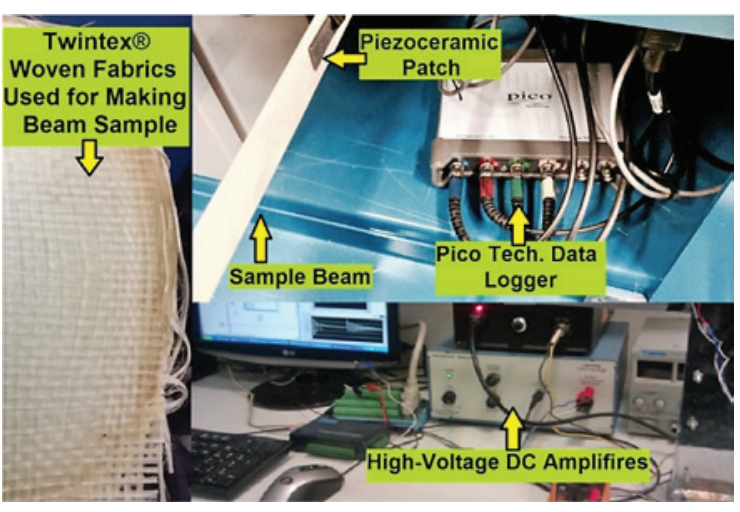

Figure 4. Experimental Setup 


\subsubsection{Numerical reliability curves Vs experimental results}

A numerical algorithm for PDE-based reliability assessment has been described before. Based on the numerical procedure, two approaches are possible for numerical reliability calculation. While there is an appropriate information about stochastic variation of $\mathrm{Z}$ during the testing, one can go through a simulation process and solve the PDE differential equation to evaluate time-varying structural reliability. As a second approach, the information about the physical variations can be attained via structural health monitoring (SHM) systems, which is the main subject of current research. In the latter approach, the reliability assessment or reliability prediction is calculated utilizing an updated system information. Here, both numerical approaches have been carried out in order to make a comparison with experimental reliability testing.

Initially, a Weibull distribution for the structural stiffness and a normal distribution for random excitation are assumed to calculate an offline PDE for time-varying reliability prediction (Figure 5). In the designated Weibull distribution, the shape and scale parameters are deduced from pre-experiment tests and calculated as $\alpha=21.763$ and $\beta=0.981$ respectively. As for the second approach, SHM data utilized for updating the probability distribution of changing parameters. Figure 5 presents the results of two approaches as compared with experimental results.

In order to assess the accuracy of the proposed reliability assessment method, more than a hundred identical beam were manufactured and a hundred samples were chosen for reliability trials. All samples were subjected to same loading condition in a similar environment and the time responses of each sample are collected under $120 \mathrm{sec}-$ onds of random loading. The three-dimensional exhibition of the trials that is depicted in Figure 6 shows how some samples go over the failure criteria during the test. Experimentally calculated reliability extracted from the results of evaluation tests are also imported in Figure 5 in order to make comparisons between experimental and numerical evaluations of the method. As evident from Figure 5, the experimental result is different as compared with the other two presented curves. It should be noticed that this different shape is because of the limited number of experiments

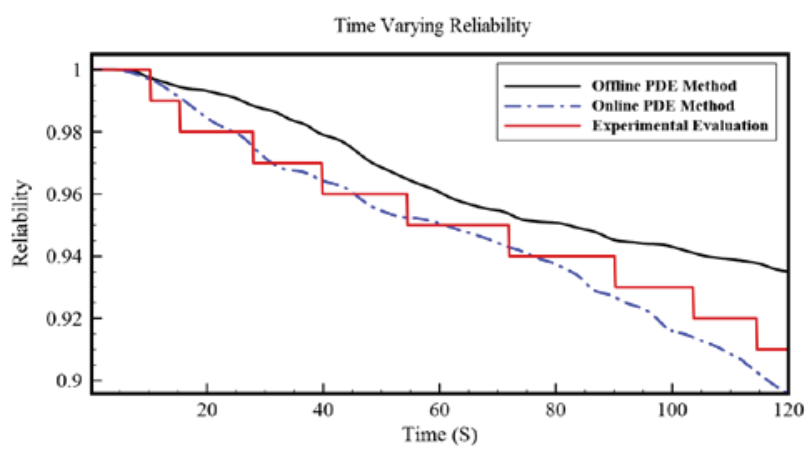

Figure 5. Experimental evaluation of PSRP method using repeated accelerated tests

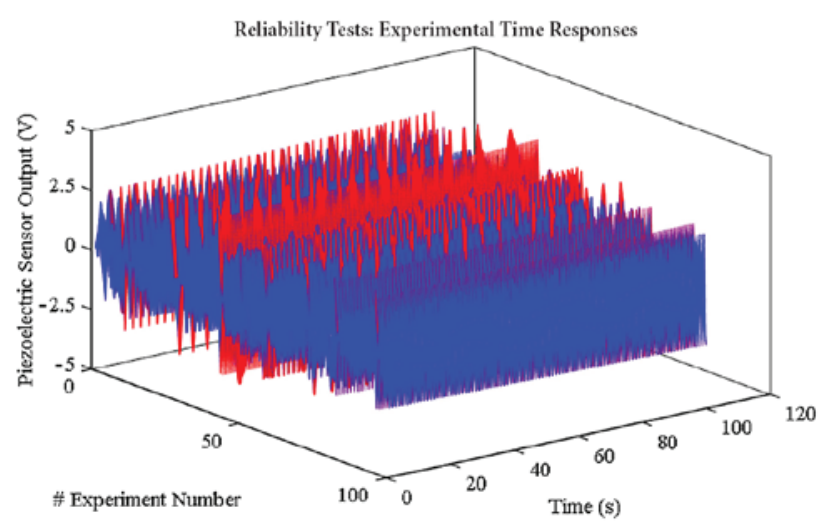

Figure 6. Time response histories during reliability evaluation trials

that results in sudden jumps in the curve while reliability decreases. Having a chance of taking more samples and thus performing more experiments, will cause the jumps to gradually get small and thus a smoother experimental curve can be concluded. However, it is evident that the presented method performs well in this experiment and the online PDE method for reliability assessment is more accurate.

\section{Wing description and stochastic measurement data}

During the previous sections, reliability calculation method is presented and evaluated experimentally. The final goal of the current research is to predict the WSC reliability using the presented PDE method. Having the structural dynamic response is the first step for utilizing PDE-based reliability method. Hence, in this section, the methodology used to predict the WSC dynamic response is illustrated. For this purpose, the WSC is augmented with piezoceramic sensors in order to obtain the required SHM data. An aircraft flexible wing is considered and modeled similarly to Figure 1 . The WSC model is divided into span-wise sections along the wing elastic axis (EA). Table 1, represents the pertinent WSC system data required for reliability analysis.

A constellation of piezoceramic sensors is also assumed to be integrated within each lower panel of the WSC sections for online SHM. The sensor input-output relation is calculated via a novel Explicit Hybrid Stabilization (EHS) technique (Hosseini Kordkheili, Salmani, \&

Table 1. WSC system data

\begin{tabular}{|l|c|}
\hline \multicolumn{1}{|c|}{ WSC Description } & Values \\
\hline Material & Aluminum 6061-T6 \\
\hline Wing Area & $159 \mathrm{~m} 2$ \\
\hline Wing Span & $25 \mathrm{~m}$ \\
\hline Thickness & $0.01 \mathrm{~m}$ \\
\hline First Bending Frequency & $1.9 \mathrm{~Hz}$ \\
\hline
\end{tabular}


Afshari, 2016) and the detailed Finite Element Piezolaminated Plate (FEPP) model of WSC used for modeling of the piezoceramic sensor responses is shown in Figure 7. For finite element formulation, the displacement of the element can be evaluated using the nodal DOFs.

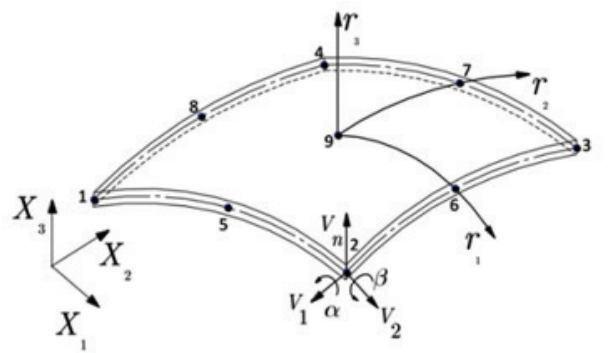

Figure 7. Degenerated shell element configuration

(Hosseini Kordkheili et al., 2016)

Now, using in-plane strain tensor, and its decomposition to the membrane and bending strains, the covariant strain tensors can be calculated in the local physical coordinate (Hosseini Kordkheili et al., 2016). In order to attain SHM data, the electromechanical behavior of the piezo-laminated shell is simulated using the work done by external mechanical forces $F_{q}$ and electric charges $F_{\phi}$ as:

$$
W=\{q\}^{T}\left\{F_{q}\right\}+\{\phi\}^{T}\left\{F_{\phi}\right\} .
$$

Where due to Hamilton's variational principle,

$$
\delta(T-S+W)=0 \text {. }
$$

Finite element formulation of equations of motion is extracted as:

$$
\left[\begin{array}{c}
{[M]} \\
{[0]}
\end{array}\right]\left\{\begin{array}{l}
\ddot{q} \\
\ddot{\phi}
\end{array}\right\}+\left[\begin{array}{cc}
{\left[K_{M}\right]} & {\left[K_{M E}\right]} \\
{\left[K_{M E}\right]^{T}} & {\left[K_{E}\right]}
\end{array}\right]\left\{\begin{array}{l}
q \\
\phi
\end{array}\right\}=\left\{\begin{array}{c}
F_{q} \\
F_{\phi}
\end{array}\right\},
$$

where $M, K_{M}, K_{M E}, K_{E}$ are the element's mass, mechanical stiffness, electromechanical stiffness and electrical stiffness matrices, respectively.

Considering above equations, via simulating the measured piezo-sensors output voltages, one can extract the WSC displacements using the reverse equations of motion (Hosseini Kordkheili et al., 2016). Basic characteristics of the piezo-sensor used in this study are presented in Table 2.

Table 2. Piezo-sensor material properties

\begin{tabular}{|l|c|}
\hline \multicolumn{1}{|c|}{ Material Properties } & PZT G1195 Piezoceramic \\
\hline \multicolumn{1}{|c|}{ Elastic Properties } & \\
\hline$E_{11}(\mathrm{GPa})$ & 63 \\
\hline$E_{22}(\mathrm{GPa})$ & 63 \\
\hline$v_{12}$ & 0.3 \\
\hline$G_{12}(\mathrm{GPa})$ & 24.2 \\
\hline$\rho\left(\mathrm{Kg} / \mathrm{m}^{-3}\right)$ & 7600 \\
\hline Piezoelectric Properties & \\
\hline$d_{31}\left(10^{-10} \mathrm{mV}^{-1}\right)$ & 2.54 \\
\hline$d_{32}\left(10^{-10} \mathrm{mV}^{-1}\right)$ & 2.54 \\
\hline$\epsilon_{33}\left(10^{-9} \mathrm{Fm}^{-1}\right)$ & 15.0 \\
\hline
\end{tabular}

\section{Stochastic modelling of CAT}

In the previous sections, the reliability calculation method is presented and consequently, the analytical way for analysing dynamic structural response is developed. In this section, we introduce the atmospheric turbulence model that is as0sumed as the main excitation force for the WSC in this study. Atmospheric turbulence is a random process that represents the air chaotic motion encountered by the aircraft in cruise flights and is referred to as Clean Air Turbulence (CAT) in the pertinent literature. The effect of CAT on the aircraft dynamic behaviour can be introduced in a variety of approaches based on the intended application. In this study, the CAT influence is superimposed on the WSC heaving equations of motion via equivalent excitation forces as a disturbed gust loading. For simulation purposes, the CAT velocity components are usually extracted from its PSD via some shaping filters whose input can be a white noise random process. The Dryden power spectral densities (PSD) form of CAT is given as:

$$
\begin{aligned}
& P_{u_{g}}(\Omega)=\sigma_{u}^{2} \frac{2 L_{u}}{\pi} \frac{1}{1+\left(L_{u} \Omega\right)^{2}} ; \\
& P_{v_{g}}(\Omega)=\sigma_{v}^{2} \frac{L_{v}}{\pi} \frac{1+3\left(L_{v} \Omega\right)^{2}}{\left[1+\left(L_{v} \Omega\right)^{2}\right]^{2}} ; \\
& P_{w_{g}}(\Omega)=\sigma_{w}^{2} \frac{L_{w}}{\pi} \frac{1+3\left(L_{w} \Omega\right)^{2}}{\left[1+\left(L_{w} \Omega\right)^{2}\right]^{2}},
\end{aligned}
$$

where $\Omega$ is the wave number that is the ratio of temporal frequency $\omega$ to the true airspeed V according to Taylor's hypothesis.

$$
\omega=\Omega V \text {. }
$$

Mathematically speaking, CAT is a Gaussian stationary Ergodic random process (Hoblit, 1988). Assuming a cursing level flight at an altitude of $35000 \mathrm{ft}$, the gust intensity in the vertical direction will be $\sigma_{w}=4.67 \mathrm{FPS}$, where the other defining Dryden-based CAT parameters are $\mathrm{L}_{\mathrm{u}}=\mathrm{L}_{\mathrm{v}}=\mathrm{L}_{\mathrm{w}}=1750 \mathrm{ft}$. Consequently, the other direction intensities will also be:

$$
\frac{\sigma_{\mathrm{u}}^{2}}{\mathrm{~L}_{\mathrm{u}}}=\frac{\sigma_{\mathrm{v}}^{2}}{\mathrm{~L}_{\mathrm{v}}}=\frac{\sigma_{\mathrm{w}}^{2}}{\mathrm{~L}_{\mathrm{w}}}
$$

In this context, the shaping filters $G(s)$, are designed to produce the proper PSD using a white noise process. Shaping filters are usually determined for the three gust velocity components as follows:

$$
\begin{gathered}
\mathrm{G}_{\mathrm{u}_{g}}(\mathrm{~s})=\frac{\mathrm{a}_{1}}{\mathrm{~s}+\mathrm{b}_{1}} \text { where } \mathrm{a}_{1}=\sigma_{\mathrm{u}} \mathrm{U}_{1} \sqrt{\frac{2}{\pi \mathrm{L}_{\mathrm{u}}}} ; \mathrm{b}_{1}=\frac{\mathrm{U}_{1}}{\mathrm{~L}_{\mathrm{u}}} \\
\mathrm{G}_{\mathrm{v}_{\mathrm{g}}}(\mathrm{s})=\frac{\mathrm{a}_{2} \mathrm{~s}+\mathrm{b}_{2}}{\mathrm{c}_{2} \mathrm{~s}^{2}+\mathrm{d}_{2} \mathrm{~s}+\mathrm{e}_{2}} \text { where } \\
\mathrm{a}_{2}= \pm \sigma_{\mathrm{v}} \mathrm{L}_{\mathrm{v}} \mathrm{U}_{1} \sqrt{\frac{3 \mathrm{~L}_{\mathrm{v}}^{3}}{\pi}} ; \mathrm{b}_{2}= \pm \sigma_{\mathrm{v}} \mathrm{U}_{1}^{2} \sqrt{\frac{\mathrm{L}_{\mathrm{v}}}{\pi}} \\
\mathrm{e}_{2}= \pm \mathrm{U}_{1}^{2} ; \quad \mathrm{c}_{2}= \pm \mathrm{L}_{\mathrm{v}}^{2} ; \quad \mathrm{d}_{2}=4 \mathrm{U}_{1}^{2} \mathrm{~L}_{\mathrm{v}}^{2}
\end{gathered}
$$




$$
\begin{aligned}
& G_{w_{g}}(s)=\frac{a_{3} s+b_{3}}{c_{3} s^{2}+d_{3} s+e_{3}} \text { where } \\
& a_{3}=\sigma_{w} L_{w} U_{1} \sqrt{\frac{3 L_{w}^{3}}{\pi}} ; \quad b_{3}=\sigma_{w} U_{1}^{2} \sqrt{\frac{L_{w}}{\pi}} ; \\
& e_{3}=U_{1}^{2} ; \quad c_{3}=L_{w}^{2} ; \quad d_{3}=4 U_{1}^{2} L_{w}^{2} .
\end{aligned}
$$

Subsequently, the stochastic sample gust histories are generated by application of white noise process as an input to the CAT filters $\mathrm{G}_{\mathrm{u}_{\mathrm{g}}}(\mathrm{s}), \mathrm{G}_{\mathrm{v}_{\mathrm{g}}}(\mathrm{s})$ and $\mathrm{G}_{\mathrm{w}_{\mathrm{g}}}(\mathrm{s})$ as shown in Figure 8.
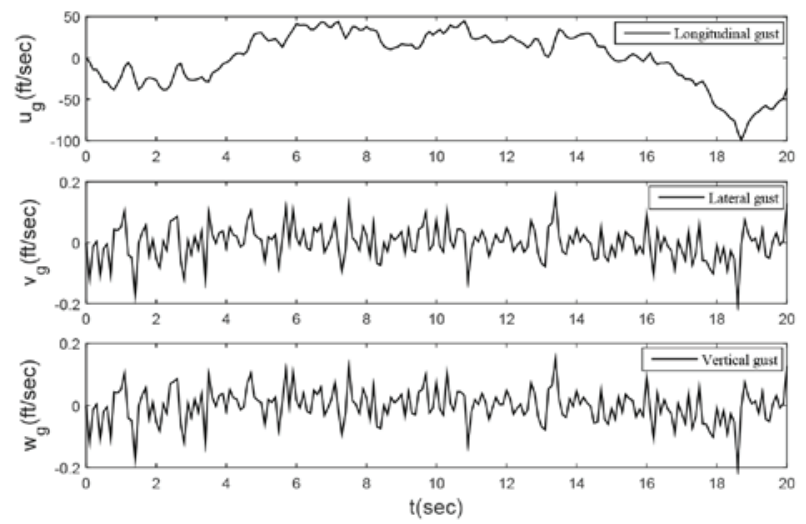

Figure 8. Turbulence velocity components during cruise flight

Subsequently, the structural response of a WSC under the action of combined stochastic gust and unsteady aerodynamics loadings should be deliberated for reliability analysis. The Theodorsen function is used to model the unsteady aerodynamic loading. In addition, since the bending resistance reliability is of interest, only the vertical heaving motion is examined. Accordingly, the WSC governing equation of motion at the center of each segment will be (Qi \& Tian, 2013):

$$
m \ddot{h}+d_{h} \dot{h}+k_{h} h=-L_{a}(t)-L_{g}(t),
$$

where $\mathrm{m}$ is the mass of each segment and $h$ corresponds to WSC heaving displacement (down is positive). Using the reduced frequency defined as $k=\frac{\omega b}{V}$. where $\mathrm{b}$ is usually taken as the mean geometric chord, we can model the loadings via the two-dimensional incompressible unsteady aerodynamic theory.

$$
L_{a}(t)=\frac{1}{2} \pi \rho_{a} b S \omega^{2} k_{a}(k) h,
$$

where,

$$
\begin{aligned}
& k_{a}(k)=-1+i 2 C(k) \frac{1}{k} \\
& L_{g}(t)=q_{d} \frac{S}{V} C_{L W}(k) \omega_{g}(t)
\end{aligned}
$$

with,

$$
C_{L W}(k)=2 \pi\left\{C(k)\left(\left(J_{0}(k)-i J_{1}(k)\right)+i J_{1}(k)\right\},\right.
$$

Where $J_{0}$ and $J_{1}$ are 0 -order and 1-order Bessel functions, respectively.
Figure 9, demonstrates the power spectral density (PSD) of the combined loadings exerted at the center of each wing segment. This excitation will next be used for numerical investigations of WSC reliability in the subsequent sections.

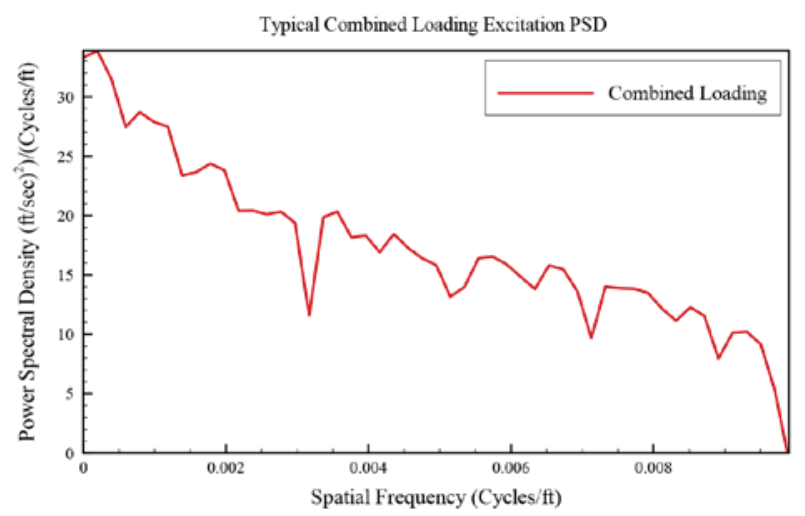

Figure 9. Power spectral density of the combined loadings

\section{Reliability of the WSC}

A stochastic shear WSC model with three different damage scenarios is investigated to evaluate the reliability of the wing under stochastic turbulence excitation. Scenarios 1 and 2 have single damage in the third wing segment with different severities of $10 \%$ and $20 \%$, respectively. Scenario 3 is a multi-damage case with deficiencies introduced in the third and sixth segments with damage severities of $20 \%$ and $10 \%$, respectively.

For a simulation time span of 20.000 hours in service cruise flight, the combined stochastic loading of Figure 8 , is exerted on the WSC. Table 3 , presents the dynamic span-wise response characteristics of the healthy and dam-

\begin{tabular}{|c|c|c|c|c|}
\hline \multirow[b]{2}{*}{ Scenario } & \multirow{2}{*}{$\begin{array}{l}\text { Frequency } \\
\quad(\mathrm{Hz})\end{array}$} & \multicolumn{3}{|c|}{ Mode (Shape) Deflection } \\
\hline & & $\begin{array}{l}\text { Root Chord } \\
\text { Section }\end{array}$ & $\begin{array}{c}\text { Mid Span } \\
\text { Section }\end{array}$ & $\begin{array}{l}\text { Wing Tip } \\
\text { Section }\end{array}$ \\
\hline Healthy & $\begin{array}{c}1.9172 \\
4.0976 \\
10.6538\end{array}$ & $\begin{array}{l}1.000 \\
1.000 \\
1.000\end{array}$ & $\begin{array}{c}13.803 \\
12.1415 \\
-0.0845\end{array}$ & $\begin{array}{c}29.17575 \\
-8.76925 \\
0.0055\end{array}$ \\
\hline Scenario 1 & $\begin{array}{c}1.8386 \\
3.9296 \\
10.2116\end{array}$ & $\begin{array}{l}1.000 \\
1.000 \\
1.000\end{array}$ & $\begin{array}{c}15.26925 \\
13.4745 \\
-0.07575\end{array}$ & $\begin{array}{c}29.93223 \\
-10.364 \\
0.005075\end{array}$ \\
\hline Scenario 2 & $\begin{array}{l}1.7708 \\
3.7846 \\
9.8346\end{array}$ & $\begin{array}{l}1.000 \\
1.000 \\
1.000\end{array}$ & $\begin{array}{c}17.11225 \\
15.139 \\
-0.06725\end{array}$ & $\begin{array}{c}31.3788 \\
-12.43 \\
0.00045\end{array}$ \\
\hline Scenario 3 & $\begin{array}{c}1.8936 \\
4.047 \\
10.5164\end{array}$ & $\begin{array}{l}1.000 \\
1.000 \\
1.000\end{array}$ & $\begin{array}{c}11.163 \\
9.61475 \\
-0.1065\end{array}$ & $\begin{array}{c}24.75975 \\
-6.559 \\
0.007825\end{array}$ \\
\hline
\end{tabular}
aged WSC in terms of the first 3 natural frequencies and their corresponding mode shapes. It should be noted that the three numbers in front of each frequency, characterize the mode shape by representing the deflection ratio at the WSC middle and tip location over the root displacement, assuming the root section deflection is $\mathrm{z}=1$.

Table 3. Dynamic Properties of the considered scenarios 
In this research, both structure and external loading are assumed uncertain. In this respect, with a similar approach as Zhang, Xu and Li (2011), the first modal damping ratio $\left(\xi_{1}\right)$ and the peak gust intensity $\left(\sigma_{\mathrm{w}_{\max }}\right)$ of the turbulence excitation are considered as random parameters or stochastic forces. Selection of these parameters could be based on some knowledge about the system uncertainties that could be available via either a pre-design experimentation or using handbooks on material properties. The probabilistic descriptions of the two random parameters taken in our study are presented in Table 4.

Table 4. Probabilistic description of the random parameters

\begin{tabular}{|l|c|c|c|}
\hline $\begin{array}{c}\text { Random } \\
\text { Parameter }\end{array}$ & Distribution & Mean & COV \\
\hline$\xi_{1}$ & Lognormal & 0.01 & 0.1 \\
\hline$\sigma_{\mathrm{w}_{\max }}$ & Normal & 4.67 & 0.5 \\
\hline
\end{tabular}

\subsection{Analysis of the WSC reliability}

According to the stress-strength theory, wing stress during the flight, cannot exceed the yield strength in order to avoid structural failures $\left(\sigma_{\max }>\sigma_{\mathrm{s}}\right)$. In this section, the WSC reliability analysis results are presented. For this purpose, the stochastic parameter vector $Z=(\Theta, \Psi)$ is first discretized into representative points $z_{\mathrm{i}}=\left(\theta_{\mathrm{q}}, \psi_{\mathrm{r}}\right), \mathrm{i}=1$, $2, \ldots, \mathrm{N}_{\mathrm{z}}, \mathrm{q}=1,2, \ldots, \mathrm{N}_{\theta}, \mathrm{r}=1,2, \ldots, \mathrm{N}_{\psi}$ in the domain $\Omega_{Z}$. Here, $\Theta$ represents the first modal damping ratio whose uncertainty is considered in the SHM process and $\Psi$ is the random parameter related to the maximum gust intensity. For the numerical investigation, the random parameter vector $\mathrm{Z}$ is discretized into representative points as depicted in Figure 10.

Following the procedure outlined in Section 2.2, WSC reliability of the healthy and the damaged structure are calculated for a time duration of 20.000 hours, whose detailed results for different scenarios are shown in Figure 11 and summarized in Table 5. As seen from Table 5, the reliability of the healthy structure is the highest for all thresholds considered, that is to be expected. Moreover,

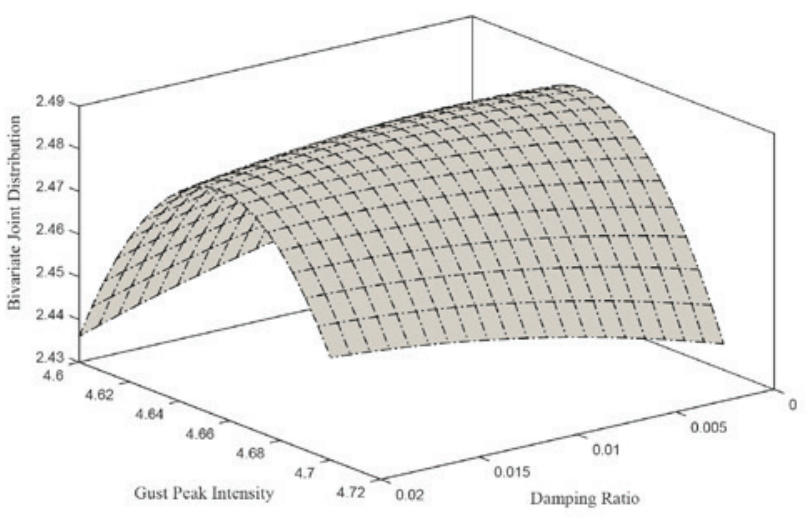

Figure 10. Stochastic parameterized domain for larger damage severity in the third WSC segment (scenario 2) a lower reliability has resulted in the damaged structure. This could serve as a theoretical verification of the presented reliability assessment procedure.

An interesting observation also appears in Table 5 that indicates a higher reliability for a more intensively damaged WSC (Scenario 3) in comparison with the second damaged scenario. This outcome can be justified by investigation of the WSC modal characteristics as well as the excitation signal. As noted from Figure 9, the PSD of the loading decreases with spatial frequency. Assuming an airspeed of 850 FPS, one can conclude that the combined excitation PSD almost vanishes for frequencies larger than $8 \mathrm{~Hz}$. On the other hand, judging by the modal results of Table 3, the natural frequencies of the first three modes in the second scenario is lower as compared with those of the healthy WSC and the other two damaged scenarios (one and three). As a result, the WSC will be more prone to the defined failure in the second scenario as compared with the third scenario. Accordingly, the WSC should, show a lower reliability in the second scenario, that is exactly what has been resulted. This can also be considered as a verification of the processes proposed for the WSC reliability assessment. Finally, it can be concluded that WSC reliability is a function of PSD of the external excitation as well as the selected thresholds for physical uncertainties. The WSC time-varying reliability for different scenarios is shown in Figure 11.

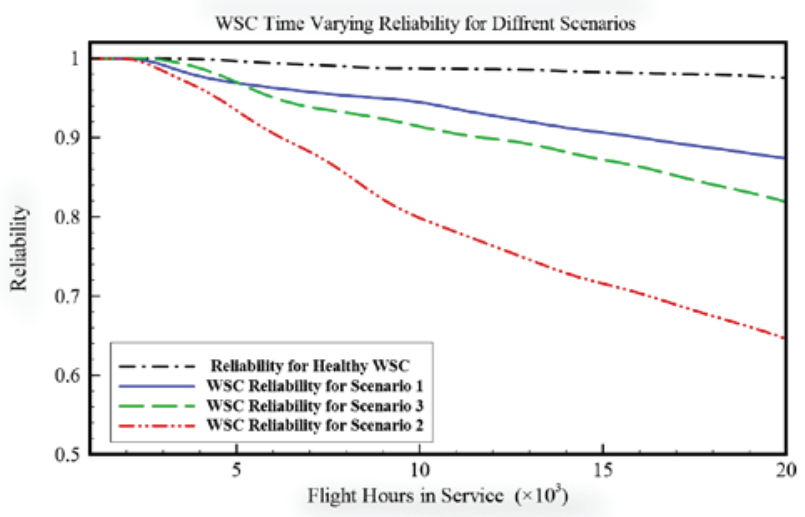

Figure 11. WSC reliability for different scenarios

Table 4. Reliability of WSC for different scenarios

\begin{tabular}{|c|c|c|c|c|}
\hline \multirow[b]{2}{*}{ Scenario } & \multicolumn{4}{|c|}{ Reliability (\%) } \\
\hline & $\begin{array}{l}\exists \\
m \\
0 \\
\vec{x} \\
n \\
11 \\
+\end{array}$ & 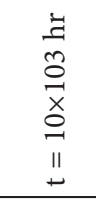 & 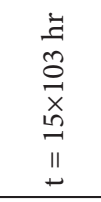 & 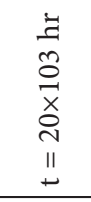 \\
\hline Healthy & 99.670 & 98.726 & 98.238 & 97.572 \\
\hline Scenario 1 & 96.906 & 94.452 & 90.625 & 87.442 \\
\hline Scenario 2 & 93.457 & 79.822 & 71.577 & 64.743 \\
\hline Scenario 3 & 96.999 & 91.395 & 87.221 & 81.997 \\
\hline
\end{tabular}




\section{Conclusions}

An integrated technique for SHM-based online reliability assessment of an aircraft wing structural component (WSC) is proposed under stochastic features of the structure, measurement data, turbulence, and aerodynamic excitations. Considering one limit state function, reliability analysis is performed via the utility of a stochastic system identification method together with Probability Density Evolution (PDE) equation. As an initial step, the presented method experimentally evaluated using a simple cantilevered beam structure. The experimental results, illustrated a great performance for the presented reliability assessment method, especially for the SHM-based reliability calculation. Accordingly, a WSC heaving equation of motion forced by a combined stochastic loading is assumed and the required equations for SHM data gathering and reliability analysis are presented in detail. Consequently, numerical investigations on the WSC under three damage scenarios is performed in order to achieve simulated SHM data and further reliability assessment. The calculated reliability of WSC for different damage scenarios demonstrates lower structural reliability for larger damage severity at similar locations. However, in a multi-damage (more severe) scenario, higher reliability was obtained as compared with a single damage scenario. This observation revealed the complex role of the WSC modal characteristics in structural reliability analysis especially when only one limit state is considered. This also remarks the importance of a proper selection of limit state parameter as well as limit state values. As a final step in the current research, time-varying reliability degradation of the WSC is determined and illustrated for different scenarios. The outcome of this paper paves the way for further researches towards online reliability-based failure prevention of aircraft structures using SHM data.

\section{Acknowledgements}

This research received no specific grant from any funding agency in the public, commercial, or not-for-profit sectors.

\section{References}

Belisario-Briceno, A., Zedek, S., Camps, T., François, R., Escriba, C., \& Fourniols, J. Y. (2014). SHM based on modal analysis: accelerometer and piezoelectric transducers instrumentation for civil engineering in heterogeneous structures. Paper presented at the EWSHM-7th European Workshop on Structural Health Monitoring.

Fassois, S. D., \& Sakellariou, J. S. (2009). Statistical time series methods for SHM. Encyclopedia of Structural Health Monitoring. John Wiley \& Sons, Ltd.

Grondel, S., Assaad, J., Delebarre, C., \& Moulin, E. (2004). Health monitoring of a composite wingbox structure. Ultrasonics, 42(1), 819-824. https://doi.org/10.1016/j.ultras.2004.01.058

Hoblit, F. M. (1988). Gust loads on aircraft: concepts and applications. AIAA.
Hosseini Kordkheili, S., Salmani, H., \& Afshari, S. (2016). A stabilized piezolaminated nine-nodded shell element formulation for analyzing smart structures behaviors. Mechanics of Advanced Materials and Structures, 23(2), 187-194. https://doi.org/10.1080/15376494.2014.949925

Li, J. (2016). Probability density evolution method: background, significance and recent developments. Probabilistic Engineering Mechanics, 44, 111-117. https://doi.org/10.1016/j.probengmech.2015.09.013

Li, J., \& Chen, J. (2004). Probability density evolution method for dynamic response analysis of structures with uncertain parameters. Computational Mechanics, 34(5), 400-409. https://doi.org/10.1007/s00466-004-0583-8

Mustapha, F., Manson, G., Pierce, S., \& Worden, K. (2005). Structural health monitoring of an annular component using a statistical approach. Strain, 41(3), 117-127. https://doi.org/10.1111/j.1475-1305.2005.00207.x

Nobahari, H., Kordkheili, S. A. H., \& Afshari, S. S. (2014). Hardware-in-the-loop optimization of an active vibration controller in a flexible beam structure using evolutionary algorithms. Journal of Intelligent Material Systems and Structures, 25(10), 1211-1223. https://doi.org/10.1177/1045389X13502874

Okasha, N. M., Frangopol, D. M., Saydam, D., \& Salvino, L. W. (2011). Reliability analysis and damage detection in highspeed naval craft based on structural health monitoring data. Structural Health Monitoring, 10(4), 361-379.

https://doi.org/10.1177/1475921710379516

Qi, W., \& Tian, S. (2013). Complex modal pseudo-excitation method for gust responses of wing structures. Paper presented at the Mechatronic Sciences, Electric Engineering and Computer (MEC), Proceedings 2013 International Conference on.

Raghavan, A., \& Cesnik, C. E. (2007). Review of guided-wave structural health monitoring. Shock and Vibration Digest, 39(2), 91-116. https://doi.org/10.1177/0583102406075428

Raouf, N., \& Pourtakdoust, S. H. (2017). Time-varying structural reliability of launch vehicle via extreme response approach. Journal of Spacecraft and Rockets, 54, 306-314. https://doi.org/10.2514/1.A33550

Saraygord Afshari, S., \& Pourtakdoust, S. H. (2017). Reliability-based optimization of an active vibration controller using evolutionary algorithms. Paper presented at the SPIE Smart Structures and Materials + Nondestructive Evaluation and Health Monitoring.

Saraygord Afshari, S., \& Pourtakdoust, S. H. (2018). Utility of probability density evolution method for experimental reliability-based active vibration control. Structural Control and Health Monitoring, 25(8), e2199. https://doi.org/10.1002/stc.2199

Soliman, M., Barone, G., \& Frangopol, D. M. (2015). Fatigue reliability and service life prediction of aluminum naval ship details based on monitoring data. Structural Health Monitoring, 14(1), 3-19. https://doi.org/10.1177/1475921714546059

Trendafilova, I., Cartmell, M., \& Ostachowicz, W. (2008). Vibration-based damage detection in an aircraft wing scaled model using principal component analysis and pattern recognition. Journal of Sound and Vibration, 313(3), 560-566. https://doi.org/10.1016/j.jsv.2007.12.008

Tuegel, E. J., Bell, R. P., Berens, A. P., Brussat, T., Cardinal, J. W., Gallagher, J. P., \& Rudd, J. (2013). Aircraft structural reliability and risk analysis handbook (Vol. 1: Basic Analysis Methods). Retrieved from http://www.dtic.mil/dtic/tr/fulltext/u2/ a587824.pdf 
Xu, Y., Zhang, J., Li, J., \& Xia, Y. (2009). Experimental investigation on statistical moment-based structural damage detection method. Structural Health Monitoring, 8(6), 555-571.

https://doi.org/10.1177/1475921709341011

Zhang, J., Xu, Y., Xia, Y., \& Li, J. (2008). A new statistical moment-based structural damage detection method. Structural Engineering and Mechanics, 30(4), 445-466.

https://doi.org/10.12989/sem.2008.30.4.445

Zhang, J., Xu, Y. L., \& Li, J. (2011). Integrated system identification and reliability evaluation of stochastic building structures. Probabilistic Engineering Mechanics, 26(4), 528-538. https://doi.org/10.1016/j.probengmech.2011.04.002

Zhu, S. P., Huang, H. Z., Peng, W., Wang, H. K., \& Mahadevan, S. (2016). Probabilistic Physics of Failure-based framework for fatigue life prediction of aircraft gas turbine discs under uncertainty. Reliability Engineering \& System Safety, 146, 1-12. https://doi.org/10.1016/j.ress.2015.10.002

\begin{tabular}{|l|l|}
\hline \multicolumn{2}{|l|}{ Nomenclature } \\
\hline $\mathbf{X}$ & Displacement vector of N order \\
\hline $\mathrm{g}(\Psi, \mathrm{t})$ & Random or deterministic external excitation \\
\hline$\Psi$ & $\begin{array}{l}n_{\Psi} \text { order random parameter vector with the } \\
\text { known probability density function }\end{array}$ \\
\hline$\Theta$ & $\begin{array}{l}\text { Random parameter vector reflecting SI } \\
\text { uncertainties }\end{array}$ \\
\hline$\phi_{i j}$ & $\begin{array}{l}i \text {-th component of the } \text {-th theoretical mode } \\
\text { shape }\end{array}$ \\
\hline$\omega_{j}(\mathbf{K})$ & $j$-th theoretical natural frequency \\
\hline$\xi_{j}$ & $j$-th modal damping ratio \\
\hline$M_{4 \theta i}$ & $\begin{array}{l}\text { Theoretical fourth-order statistical moments of } \\
\text { the } i \text {-th-story drift }\end{array}$ \\
\hline$\hat{\mathbf{M}}_{4}$ & $\begin{array}{l}\text { Measured fourth-order moment vector of story } \\
\text { drifts of the WSC }\end{array}$ \\
\hline $\mathrm{P}_{\mathrm{XZ}}(\mathrm{x}, \mathrm{z}, \mathrm{t})$ & $\begin{array}{l}\text { Joint Probability Density Function (PDF) of } \\
\text { X(t) and the random parameter vector Z }\end{array}$ \\
\hline $\mathrm{u}_{\mathrm{g}}, \mathrm{v}_{\mathrm{g}}, \mathrm{w}_{\mathrm{g}}$ & Gust velocities in the X, Y and Z directions \\
\hline $\mathrm{L}_{\mathrm{u}}, \mathrm{L}_{\mathrm{v}}, \mathrm{L}_{\mathrm{w}}$ & Turbulence scales \\
\hline$\sigma_{\mathrm{u}}, \sigma_{\mathrm{v}}, \sigma_{\mathrm{w}}$ & Gust Intensities \\
\hline $\mathrm{C}(\mathrm{k})$ & Theodorsen function \\
\hline $\mathrm{S}$ & Projected area of the desired wing segment \\
\hline$d_{h}$ & Damping coefficient of the segment \\
\hline$k_{h}$ & Stiffness coefficient of the segment \\
\hline$L_{a}$ & $\begin{array}{l}\text { Aerodynamic lift } \\
\text { nodal DOFs }\end{array}$ \\
\hline$L_{g}$ & Electric potential of the piezoelectric layer \\
\hline$\left(x_{1}^{k}, x_{2}^{k}, x_{3}^{k}\right)$ & Finite element nodal coordinates \\
\hline$\left\{q_{j}\right\}$ & \\
\hline$\phi_{k}$ & \\
\hline
\end{tabular}

